\title{
Pulmonary perfusion imaging using MRI: clinical application
}

\author{
Sebastian Ley • Julia Ley-Zaporozhan
}

Received: 24 May 2011 / Accepted: 16 November 2011 / Published online: 29 December 2011

(C) European Society of Radiology 2011

\begin{abstract}
Background Lung perfusion is one of the key components of oxygenation. It is hampered in pulmonary arterial diseases and secondary due to parenchymal diseases.

Methods Assessment is frequently required during the workup of a patient for either of these disease categories. Results This review provides insight into imaging techniques, qualitative and quantitative evaluation, and focuses on clinical application of MR perfusion.

Conclusion The two major techniques, non-contrast-enhanced (arterial spin labeling) and contrast-enhanced perfusion techniques, are discussed.
\end{abstract}

Keywords Magnetic resonance imaging · Perfusion · Lung · Application - Arterial spin labeling perfusion .

Contrast-enhanced perfusion

\section{Introduction}

Perfusion of the lung is one of the main components required for pulmonary gas exchange. Pulmonary perfusion is altered in physiological and pathological conditions such as

S. Ley $(\bowtie) \cdot J$. Ley-Zaporozhan

Division of Cardiothoracic Imaging, Department of Medical

Imaging, Toronto General Hospital, University of Toronto and

University Health Network, Toronto General Hospital,

585 University Avenue,

Toronto, Ontario M5G 2N2, Canada

e-mail: ley@gmx.de

\section{S. Ley}

Department of Diagnostic and Interventional Radiology,

University Hospital Heidelberg,

Im Neuenheimer Feld 430,

69120 Heidelberg, Germany pulmonary embolism, pulmonary hypertension and chronic obstructive disease. Therefore, assessment of the pulmonary perfusion is of physiological and clinical importance. As the lung is a large, diffuse and inhomogeneous organ it can only be studied in detail with techniques providing good to excellent spatial resolution. One of these techniques is magnetic resonance imaging (MRI). For more than 20 years MRI has been available as a technique for assessment of pulmonary perfusion [1]. Two principal techniques are established: arterial spin-labeling and contrast-enhanced perfusion [2]. Especially the tremendous technical developments of the recents years regarding hardware and new sequences make MR-based perfusion imaging broadly available. Therefore, this review article will elucidate the current state of the art sequences, clinical applications and future developments of each technique.

\section{Non-contrast-enhanced pulmonary perfusion}

Technique

MR offers the possibility to mark a specific part of spins magnetically by selective radiofrequency (RF) excitation, leading to a specific magnetisation to a selected fraction of blood. This technique is called arterial spin labeling (ASL) and requires no intravenous application of contrast material and is therefore considered to be completely non invasive [3].

It has the advantage that measurements can be made repeatedly over very short time periods (seconds). The absence of contrast also means that the measurements can be repeated indefinitely as it has not to be cleared out from the blood before reimaging is possible (also, the absolute number of measurements is limited by the total amount of 
contrast material that can be applied in 1 day). There are some limitations of these techniques: (1) more difficult to implement compared with dynamic contrast-enhanced imaging; (2) requires a regular cardiac cycle; (3) single slice technique and 5-8 slices have to be acquired to cover the whole lung; (4) the limited voxel resolution (i.e. $5 \times 5 \times 30 \mathrm{~mm}^{3}$ ) [4].

In essence, two ECG-gated images of a selected slice are taken 5-8 s apart during a single apnea. The two images differ only in the way that tagging radiofrequency (RF) pulses change the signal of blood flowing into the imaged section while keeping the signal from the stationary structures unchanged between images. Subtraction of the two images leaves an image in which the signal from a volume element (voxel) is proportional to the amount of pulmonary arterial blood delivered during the previous heart cycle [3, 5-11]. In principle, ASL preparation can be combined with many fast data recording techniques. True fast imaging with steady precession (true FISP) sequences in particular has been found to be ideally suited for assessment of pulmonary perfusion.

Specific types of ASL techniques that allow for quantification of regional pulmonary perfusion during a single breath hold are called "Flow-sensitive Alternating Inversion Recovery" (FAIR) and "Flow-sensitive Alternating Inversion Recovery with an Extra Radio frequency (RF) pulse" (FAIRER). Detailed descriptions of the different principles are given elsewhere [4, 12]. Perfusion-weighted images are obtained from the subtraction of a control and tag images, which are interleaved and can be acquired within one breathholding period of 20-27 s (for each slice) [13]. The potential sources of artefacts are cardiac and respiratory motion. Cardiac motion and pulsatile flow of the descending aorta can generate smearing and flow artefacts, respectively. Spatial variation between the control images can lead to ghosting of the blood vessels, and spatial movement between the control and tag images causes the appearance of the adjacent dark-bright pairs of the blood vessels.

A closely related technique, called the T1 method, has also been used to measure pulmonary perfusion (Fig. 1) [14, 15]. Methodologically, this technique is similar to the ASL techniques. It differs primarily in how the experiment is conceptualised - as the delivery of a bolus of tagged spins or as an apparent alteration of the longitudinal relaxation time. Perfusion-weighted images are acquired in a single shot by completely saturating the static background tissue before image acquisition. The decrease of the lung $\mathrm{T} 1$ values in the same slice position from the global to the selective experiment is statistically significant and results from the wash-in effect of the noninverted blood water ${ }^{1} \mathrm{H}$ spins outside the imaging slice. Because the perfusion-weighted image is acquired in a single shot, artefacts arising from patient motion or different respiratory levels can be avoided.

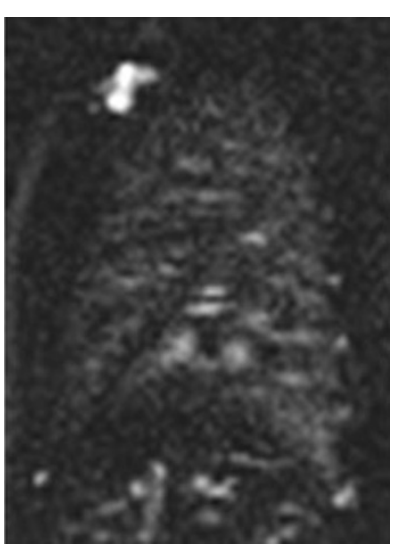

Fig. 1 Arterial spin labeling (ASL) image in a healthy volunteer (sagittal orientation, right lung). The spatial resolution is $3.9 \times 3.9 \times$ $10 \mathrm{~mm}$, which allows for visualisation of the major and minor fissure. On the other hand the signal-to-noise ratio is low, making an detailed evaluation difficult

Furthermore, only very short acquisition times $(\leq 5 \mathrm{~s}$ per slice) are required for the perfusion-weighted image, facilitating rapid and comfortable examinations. This is especially important for lung imaging, as some patients are not able to hold their breath for more than a few seconds.

\section{Quantification}

ASL-based techniques allow for calculation of relative pulmonary blood flow (rPBF) in $\mathrm{ml} / 100 \mathrm{~g} / \mathrm{min}[12,16,17]$. For conversion of rPBF into PBF the lung density is required, which is usually assumed to be constant with $0.33 \mathrm{~g} / \mathrm{ml}$ (at functional residual capacity) [18]. However, this might not be the case in lung diseases like fibrosis or emphysema. Normal values are reported to be in the range of $5.5 \mathrm{ml} / \mathrm{g} / \mathrm{min}$.

Using a rapid IR Snapshot FLASH technique, a spin labeling method within the imaging slice was used in combination with a simple two-compartment lung tissue model. The measured pulmonary perfusion rates of the healthy lung parenchyma were on the order of 400 to $600 \mathrm{ml} / 100 \mathrm{~g} / \mathrm{min}$, depending on the subject investigated and the actual slice position in the lung [19].

In healthy volunteers $(n=6)$ a quantitative comparison between non contrast-enhanced and contrast-enhanced techniques was performed [20]. Two coronal slices (middle and dorsal part of the lung) were assessed and maps of relative pulmonary blood flow (rPBF) were calculated - no absolute perfusion measures were calculated. By placing ten regions of interest in each lung, a right-to-left perfusion ratio analysis was performed. For the dorsal slice the differences between both techniques were not statistically different. The middle position however showed a $16-43 \%$ underestimation of FAIR-derived $\mathrm{rPBF}$ in the right lung. This was explained by tracer saturation for the right lung as the right pulmonary artery is in the plane of both pulses needed for tagging and 
readout. In an internal control this difference was not present if slices were acquired in a sagittal orientation.

\section{Application}

Based on the strength and limitations noted above, the clinical application so far has been limited. However, the strength of the techniques lies in the ability to study physiological aspects of the lung perfusion on a regional level [21-24]. Important for all perfusion studies is to notice that the SNR of ASL images is low, particularly in the anterior portions of the lung. Therefore, positioning the patient so that the area of interest is down-gravity may increase SNR and improve visibility of perfusion defects. Also, signal intensity and subsequently perfusion increases during examination on end-expiratory breath-hold compared to endinspiratory breath-hold $[25,26]$.

Normal aging is associated with a decline in pulmonary function and efficiency of gas exchange. The effect of aging on spatial heterogeneity of pulmonary perfusion was studied on 56 healthy, non smoking volunteers with an age range of 21-76 years [27]. Relative dispersion increased significantly with increasing age by $0.1 /$ decade until age $50-59$ years, and a significant positive relationship between relative dispersion and age was found. No regional lung density measurements were performed. Therefore, it was not possible to investigate if the age-related parenchymal changes were the cause for this phenomenon. In an early study Keilholz et al. demonstrated the ability of ASL to depict the gravitydependent perfusion effects [28]. Healthy volunteers were placed inside the scanner on their right and left lateral side and coronal images were acquired within a 10-s breath-hold using a FAIRER HASTE sequence. In both positions, an increase in the intensity of the dependent lung was found ( $229 \%$ for left lateral, $40 \%$ for right lateral). No change was seen along an isogravitational plane. These results were confirmed for volunteers lying on their back [15].

In six rabbits a pulmonary emboli model was introduced using a balloon catheter that was placed in the left pulmonary artery [29]. After occlusion of the left pulmonary artery a set of FAIRER images followed by a contrast-enhanced angiography was performed. The huge perfusion defect (whole left lung) was depicted by both imaging techniques. It has to be noted again, that $8 \mathrm{~s}$ were required for a single slice of ASL, while the 3D acquisition of the contrastenhanced perfusion needed $30 \mathrm{~s}$. A similar experiment was done in 5 rabbits were ASL perfusion and ventilation images using hyperpolarised 3helium were assessed [30]. After occlusion of the pulmonary artery by a balloon catheter the ventilation was unchanged and the ASL perfusion images demonstrated the large perfusion defect.

In patients susceptible to high altitude pulmonary oedema (HAPE) it was possible to demonstrate the uneven hypoxic pulmonary vasoconstriction compared to non-susceptible patients by assessing the relative dispersion of blood flow [31].

Future developments

Up to now, only few reports exist on the clinical application of non contrast-enhanced perfusion techniques. Due to the lengthy acquisition times and relatively low signal increase, the clinical usage will be limited in the future. It may be the imaging technique of choice for pregnant women to rule out pulmonary emboli. However, in this stetting other native imaging techniques, like steady-state free precession sequences, also showed a good clinical potential [32]. Therefore, the main application will be in exploration of physiological processes where the possibility of unrestricted measurements outweighs the prolonged acquisition time [3].

\section{Contrast-enhanced MR perfusion}

\section{Technique}

MRI of contrast-enhanced lung perfusion is realised by rapid imaging of the first pass of contrast material through the lungs after intravenous bolus injection. Twodimensional approaches can be acquired in a fast fashion such as $0.3 \mathrm{~s}$ for each slice. On the other hand, they lack sufficient spatial resolution and anatomic coverage to characterise most of the perfusion defects or inhomogeneities. For this purpose 3D techniques are mandatory. To visualise the peak enhancement of the lungs, the temporal resolution of contrast-enhanced MRI of lung perfusion has to be below the transit time of a contrast bolus through the lungs, being in the range of 3 to $4 \mathrm{~s}$ [33]. Additionally, for clinical usage a reasonable spatial resolution and anatomic coverage are required to allow for visualisation of perfusion changes on a segmental level. The most frequently used technique is a 3D gradient echo pulse sequence (FLASH) with a short TE and TR with a resulting temporal resolution of 1.0 to $1.5 \mathrm{~s}$ for each 3D data set, dependent on the used slice thickness of $10 \mathrm{~mm}$ down to $5 \mathrm{~mm}[34,35]$. Improvements in $\mathrm{k}$-space sampling techniques such as the use of parallel imaging techniques allow for increased spatial resolution without reduction of temporal resolution. A second strategy is to use echo sharing (like TREAT), where the low frequency kspace data are updated more often than the high frequency $\mathrm{k}$-space data, which is interpolated between consecutive time frames, thus leading to an effective shortening of the total acquisition time [36]. Image quality of both techniques was assessed in nine patients [37]. Using the same temporal resolution the voxel size was decreased by $24 \%$ in the TREAT data sets compared to the standard perfusion 
technique (both techniques used parallel imaging with an acceleration factor of 2). For central and peripheral vascular perception the TREAT perfusion was rated significantly superior to the standard technique. Basic evaluation of MR perfusion was performed qualitatively in comparison to perfusion scintigraphy. Intermodality comparison showed a good agreement $(\mathrm{kappa}=0.74)$ in 14 patients with various diseases (COPD, lung cancer, etc.) [38]. A direct comparison between different $\mathrm{k}$-space acquisition techniques was performed recently [39]. Three k-space acquisition techniques were compared for image quality and SNR: (1) generalised autocalibrating partial parallel acquisition (GRAPPA) with an internal acquisition of reference lines, (2) GRAPPA with a single "external" acquisition of reference lines before the measurement, and (3) a combination of GRAPPA with an internal acquisition of reference lines and view sharing. All examinations were performed in ten healthy volunteers ( $5 \mathrm{ml}$ Gadubutrol with an injection speed of $2.5 \mathrm{ml} / \mathrm{s}$ ) with a constant voxel size: $3.9 \times 3.9 \times 5 \mathrm{~mm}^{3}$. At $1.5 \mathrm{~T}$, view sharing resulted in the best image quality and SNR. Furthermore, the temporal resolution was the shortest with $1.07 \mathrm{~s}$.

So far, most of the studies used $0.1 \mathrm{mmol} \mathrm{Gd-DTPA} / \mathrm{kg}$ bodyweight for visualisation of pulmonary perfusion. Different amounts of contrast media $(0.05 \mathrm{mmol} / \mathrm{kg}$ bodyweight and $0.1 \mathrm{mmol} / \mathrm{kg}$ bodyweight), contrast media concentrations $(0.5 \mathrm{mmol} / \mathrm{l}$ and $1 \mathrm{mmol} / \mathrm{l})$, and injection protocols $(2.5 \mathrm{ml} / \mathrm{s}$ and $5 \mathrm{ml} / \mathrm{s})$ were compared in 10 healthy volunteers [40]. By analysing the signal-to-noise ratio no difference was found between the different contrast media concentrations giving the same injection speed. For both contrast agents a dose of $0.1 \mathrm{mmol} / \mathrm{kg}$ bodyweight was superior to the half dose protocol. In combination with a rapid injection $(5 \mathrm{ml} / \mathrm{s})$ the highest SNR in all vascular segments were achieved. Regarding the contrast media injection speed, a small bolus profile and fast attenuation of the lung parenchyma is optimal. It was found that with increased speed, the mean transit time (MTT) for the pulmonary arteries decreased significantly [41]. However, this decrease was not linear: a four-fold increase in injection rate led to a two-fold decrease in MTT. In the same study it was found, that the cardiac function parameters had no significant influence on the bolus profile. Overall, an injection speed of $3 \mathrm{ml} / \mathrm{s}$ was recommended for pulmonary perfusion [42].

Basic post-processing for improvement of visualisation of perfusion is done by subtraction of the data set with the highest signal intensity from a baseline data set (Fig. 2). In most cases this allows for a rapid analysis of the clinical situation of the patient. However, for any detailed analysis, therapy monitoring or presurgical estimation of the regional pulmonary function, a quantitative analysis is important. A summary of frequently used contrast media injection regimens are provided in Table 1.
Quantification

Quantification of pulmonary perfusion is based on the indicator dilution theory where the maximum of signal intensity and the temporal course of the signal change are used. For quantification an arterial input function (AIF) has to be defined by placing a region of interest (ROI) in the main pulmonary artery. The tissue response function of the lung parenchyma can be either determined by multiple single small ROIs or, better, for the whole lung.

Assuming a linear relation between the signal and the concentration of contrast agent, signal-time curves are converted to concentration-time curves [43]. Hypothesising a negligible amount of extravasating contrast agent during the first pass of the bolus in the lungs, the principles of the indicator dilution theory [44] were applicable. The central volume theorem states the relationship between the perfusion parameters regional pulmonary blood flow (PBF ml/ $100 \mathrm{ml}$ lung tissue/min), regional pulmonary blood volume (PBV $\mathrm{ml} / 100 \mathrm{ml}$ lung tissue), and the mean transit time (MTT, s):

$M T T=\frac{P B V}{P B F}$

Regional PBV can be calculated by normalising the area under the tissue concentration-time curve to the integral of the arterial input function AIF:

$P B V=\frac{\int C(t) d t}{\int A I F(t) d t}$

In consideration of the finite length of the bolus and its dispersion during its way to the tissue volume, the relationship between the AIF and the tissue concentration-time curve is described by the convolution of the AIF and a residue function $\mathrm{R}(\mathrm{t})$ :

$C(t)=P B F \int A I F(\tau) \bullet R(t-\tau) d \tau$

where $\mathrm{R}(\mathrm{t})$ is the amount of contrast agent remaining in the tissue at time t. PBF is therefore the initial height of $R(t 0)$, respectively, the maximum of $\mathrm{R}(\mathrm{t})$ in case of a delay between the $\operatorname{AIF}(t)$ and $C(t)$, and can be assessed by deconvolving $\operatorname{AIF}(\mathrm{t})$ and $\mathrm{C}(\mathrm{t})$. The deconvolution of the last equation is performed using singular value decomposition (SVD) [45], a model-independent nonparametric deconvolution analysis with the potentiality of reducing the noise contribution by applying a suitable threshold to a singular value matrix. This method has shown reliable results for first-pass bolus tracking in clinical studies [46].

Inclusion of the central pulmonary arteries leads to a systematic overestimation of pulmonary perfusion. Therefore, the use of small ROIs in the lung periphery can be 

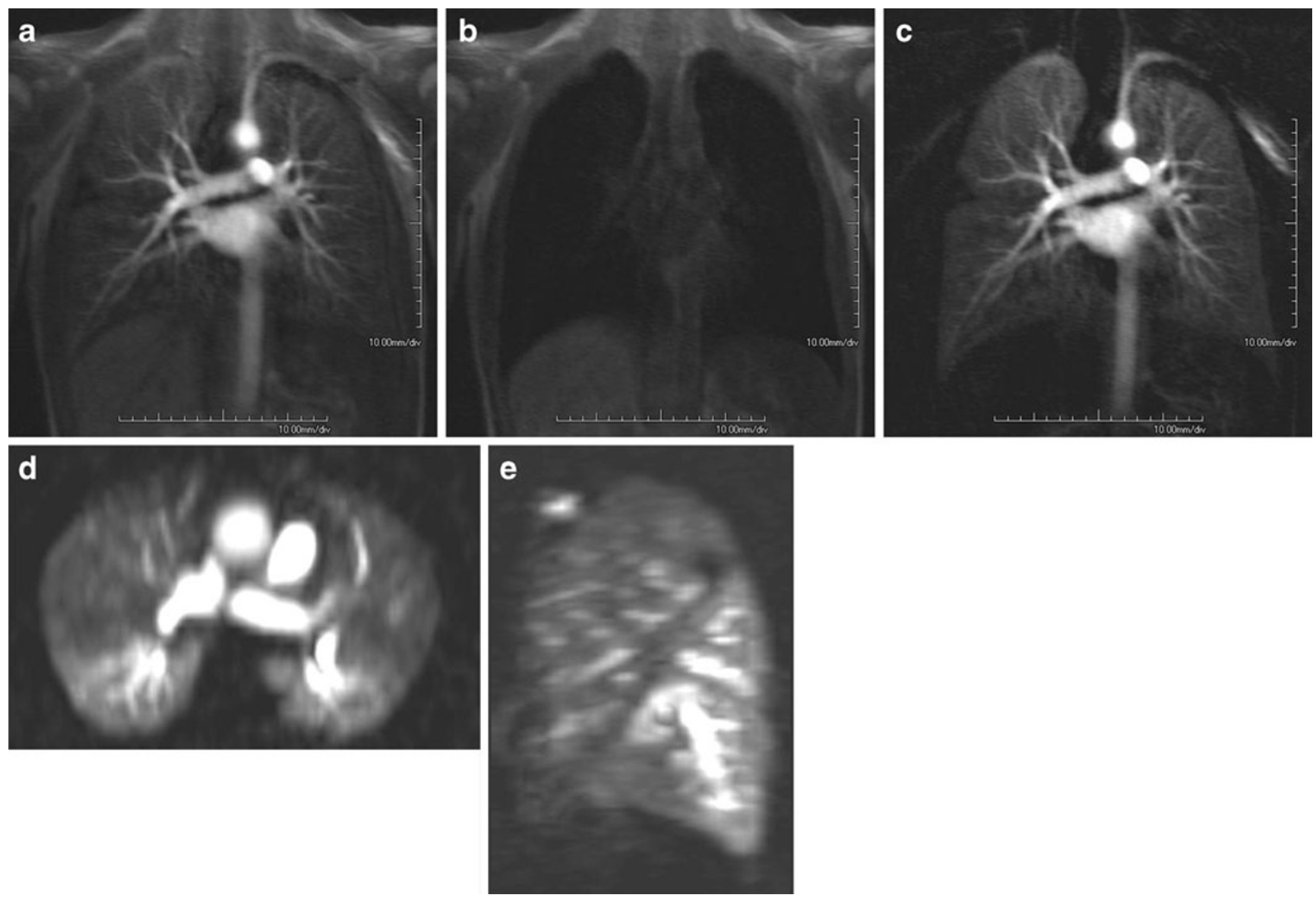

Fig. 2 Concept of subtraction of perfusion images in a healthy voluneer. (a) represents the data set with the maximum contrast enhancement. (b) is an early data set without contrast media. Subtraction of

beneficial. However, if the spatial distribution of the underlying lung disease is unknown, or if lung perfusion quantification is required for an entire lung (e.g. to assess side differences of lung perfusion) a measurement of a limited lung sample in a small ROI will only reflect a poor estimate of lung perfusion. One study used a cross-correlation analysis (CCA) of perfusion data sets for suppression of central both data sets (c) results in an image with suppressed background and therefore enhanced perfusion. The 3D nature of the data sets allow for reformation in any plane, like axial (d) or sagittal (e)

pulmonary vessels [47]. CCA has previously been used to improve the SNR and arteriovenous separation in timeresolved MR angiography [48, 49]. It was shown that it is feasible to identify vessels with a similar signal-intensity time curve as a reference vessel using CCA. For this purpose reference ROIs have to be drawn in the pulmonary artery and pulmonary vein. The approach was evaluated in
Table 1 Summary of used contrast media injection protocols and spatial resolution of perfusion-weighted MR

\begin{tabular}{llll}
\hline Injection speed $[\mathrm{ml} / \mathrm{s}]$ & Dose & Spatial resolution $\left[\mathrm{mm}^{3}\right]$ & Reference \\
\hline 2.5 & $5 \mathrm{ml}$ Gadubutrol & $3.9 \times 3.9 \times 5$ & {$[39]$} \\
5 & $5 \mathrm{ml}$ Magnevist & $1.9 \times 1.8 \times 10$ & {$[56]$} \\
3 & $0.05 \mathrm{mmol} / \mathrm{kg} \mathrm{BW}$ Magnevist & $3.9 \times 3.9 \times 6.3$ & {$[59]$} \\
4 & $0.125 \mathrm{mmol} / \mathrm{kg} \mathrm{BW}$ Magnevist & $2.9 \times 1.6 \times 10$ & {$[76]$} \\
5 & $5 \mathrm{ml} \mathrm{Omniscan}$ & $1.9 \times 1.8 \times 12$ & {$[63]$} \\
5 & $0.1 \mathrm{mmol} / \mathrm{kg} \mathrm{BW}$ Magnevist & $3.5 \times 1.9 \times 4$ & {$[65]$} \\
5 & $0.1 \mathrm{mmol} / \mathrm{kg}$ BW Magnevist & $1.9 \times 3.8 \times 3.6$ & {$[66]$} \\
5 & $0.1 \mathrm{mmol} / \mathrm{kg} \mathrm{BW}$ Multihance & $1.9 \times 3.8 \times 4$ & {$[69]$} \\
$3-5$ & $3-5 \mathrm{ml} \mathrm{Magnevist}$ & $1.9 \times 1.8 \times 10$ & {$[34]$} \\
5 & $0.1 \mathrm{mmol} / \mathrm{kg}$ BW Omniscan & $1.9 \times 3.6 \times 4$ & {$[73]$} \\
\hline
\end{tabular}


seven healthy volunteers. The quantification of PBV and $\mathrm{PBF}$ resulted in an average reduction of $25 \%$ for PBV and $15 \%$ for $\mathrm{PBF}$ compared with the segmentations without vessel exclusion. A comparison of the manual exclusion of vessel structures and the CCA revealed an average reduction of $13 \%$ for the PBV and $8 \%$ for PBF. The reduction by applying the CCA was significant $(P \leq 0.001)$ compared with the results with manual and without blood vessel exclusion for all calculated parameters.

Basically, during quantitative perfusion evaluation the signal-time curves are converted into concentration-time curves. Therefore, a linear relationship between signal intensity and concentration is mandatory. This is especially important for the arterial input function where all contrast media passes by in a compact bolus [50]. A linear relationship, however, is only seen for a small range of concentrations of 3,4 or $5 \mathrm{mmol} / \mathrm{l}$ as seen in in-vitro experiments [5153]. The linearity between signal and concentration is dependent on the sequence and parameters used (like TE). Thus, the use of a saturation recovery prepared FLASH sequence was proposed [53], which is often used in myocardial perfusion imaging, to improve the linearity of the measurements and thus the contrast-to-noise ratio (CNR) at higher doses. Applied to volunteers the usage of $0.057 \mathrm{mmol} / \mathrm{kg}$ bodyweight was feasible [53], while in an animal experiment it was found that already $0.05 \mathrm{mmol} \mathrm{Gd}-$ DTPA $/ \mathrm{kg}$ bodyweight resulted in in-vivo concentrations outside the linearity range [52]. However, comparing the quantitative measures of the $0.05 \mathrm{mmol} / \mathrm{kg}$ bodyweight the values were too high compared to results from PET where PBF was found to be $121 \mathrm{ml} / \mathrm{min} / 100 \mathrm{ml}$ [54] and PBV 17$20 \mathrm{ml} / 100 \mathrm{ml}$ [55]. In a prospective trial different injection protocols and contrast media concentrations were investigated and compared to quantitative analysis of perfusion SPECT [56]. The amount of the MR contrast bolus was fixed with $5 \mathrm{ml}$ and an injection speed of $5 \mathrm{ml} / \mathrm{s}$, followed by a $20 \mathrm{ml}$ saline flush. This approach ensured similar bolus widths in all applications. It was found that for patients weighing less than $70 \mathrm{~kg}$ a concentration of $0.3 \mathrm{mmol} / \mathrm{ml}$ showed the highest accordance to SPECT and $0.5 \mathrm{mmol} / \mathrm{ml}$ for patients weighing more than $70 \mathrm{~kg}$. However, this study used 10-mm-thick slice partitions. Therefore, the small amount of $5 \mathrm{ml}$ of contrast media resulted in an appropriate contrast-to-noise ratio.

Another solution for this complex problem was the usage of a dual bolus approach [51]. In this experiment, the arterial input function is determined by a low volume application of contrast media (i.e. $0.01 \mathrm{mmol} / \mathrm{kg}$ bodyweight) to achieve a linear relationship between contrast-agent concentration and signal intensity in the large pulmonary vessels. This is followed by boluses with higher doses to obtain sufficient CNR in lung tissue. This may allow absolute quantification as well as the visual analysis of perfusion in lung tissue, which is often not possible when a single low dose is used for absolute perfusion quantification. The drawback of this approach is the small and therefore short bolus (i.e. $1.5 \mathrm{ml}$ ) for the arterial input function, which requires an extremely high temporal resolution of the perfusion sequence to asses the peak signal. The combination of a small prebolus (0.01 $\mathrm{mmol} / \mathrm{kg}$ bodyweight) with a main bolus of $0.04 \mathrm{mmol} / \mathrm{kg}$ bodyweight resulted in perfusion parameters best comparable with literature data. A combination of the prebolus with a main bolus of $0.08 \mathrm{mmol} / \mathrm{kg}$ bodyweight resulted in an underestimation of PBV [51].

For perfusion imaging of the lung, the optimal combination of temporal resolution and contrast-to-noise ratio is essential. This complex interaction was studied using a Monte-Carlo simulation and volunteer examinations [57]. It was found that a temporal resolution of approximately $2 \mathrm{~s}$ has to be achieved for correct application of the deconvolution algorithm. The influence of noise in the data is especially relevant for the estimates of PBF: high noise levels (i.e. low CNR values) lead to a substantial underestimation and a significant loss of accuracy in the PBF estimates. It was concluded that the assessment of PBF might benefit from less aggressive acceleration, for example, by a lower acceleration factor of 2 . The CNR can be notably increased if larger voxel sizes are employed; the voxel size should thus be chosen as large as clinically acceptable.

Repeatability of quantitative perfusion measurements was tested in two patient groups and one large volunteer study. In one study ten patients with bronchioalveolar carcinoma were scanned twice within 3 days in inspiratory breath-hold [56]. Evaluation was performed by manually placing three regions of interest in the data set. An excellent agreement between both measurements was found, without any significant difference. Ten patients with pulmonary hypertension were re-scanned 3 weeks after the initial scan [58]. Three-dimensional perfusion data sets were acquired in inspiratory breath-hold and evaluation comprised the whole lung. No significant difference was found for PBF, PBV, and MTT. Fourteen volunteers were examined twice after $24 \mathrm{~h}$ in inspiratory and expiratory breath-hold [59]. Evaluation was done comprising quantitative whole lung evaluation and was performed by two observers. No significant difference was found for the pair of measurements performed in expiration and evaluated by the same observer. The inspiratory data sets however showed significant differences for PBF. Significant differences were found in inspiration and expiration for the interobserver variability. These three studies demonstrate the significant influence of the observer on the quantitative results. Therefore, for a broad usage of the technique an automatic segmentation approach assisting evaluation should be developed. Especially in data sets with pathologically changed perfusion (i.e. peripheral perfusion defects), automatic segmentation algorithms will 
be difficult to be implemented. An initial study used morphological HASTE images for segmentation of the lung borders [60]. These contours were applied to the perfusion data sets after image registration of the HASTE and perfusion images. This reduced the time needed for evaluation of the perfusion data sets to approximately $10 \mathrm{~min}$. However, this technique was only applied to data sets of healthy volunteers and no reproducibility experiments were performed.

So far MR perfusion of the lung parenchyma has been performed at $1.5 \mathrm{~T}$. Given the benefits of higher field strengths (like $3 \mathrm{~T}$ ) a higher SNR can be expected, which should be beneficial regarding quantification or which allows an increase in spatial resolution.

\section{Application}

In clinical routine, contrast-enhanced perfusion can be easily applied for visual assessment of disease characteristics. For a qualitative approach the discussion of temporal resolution and contrast media dose is obsolete and it can be performed at any MR scanner. Perfusion defects due to vascular obstruction show a typical wedge shape including the lung periphery (Fig. 3) [61]. In 41 patients with acute PE the agreement of MR perfusion with single-photon emission computed tomography (SPECT) perfusion for perfusion defects down to the subsegmental level was assessed [62]. MR perfusion showed a very high agreement with SPECT (kappa value per examination 0.98 , and $0.98,0.83$, and 0.69 for lobar, segmental, and subsegmental perfusion defects, respectively). By a quantitative segmental perfusion analysis in patients with acute pulmonary embolism it was possible to determine the obstruction index [63]. This perfusion-based index showed a higher correlation with

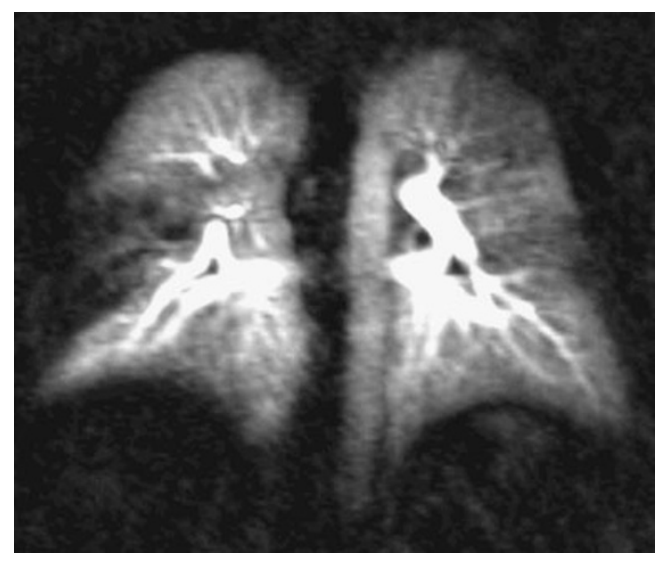

Fig. 3 Coronal 10-mm maximum intensity projection (MIP) image in a patient in a follow-up examination after acute pulmonary emboli. There is a residual occlusion of the middle lobe leading to a wedgeshaped perfusion defect on the right. There are also residual subtotal obstructions noted in the left upper and lower lobe characterised by a reduced perfusion clinical severity and also was a significant predictor of outcome. This initial report brings MR perfusion in the pole position for being discussed as new modality of choice for $\mathrm{PE}$ assessment. Furthermore, this radiation-free technique is optimally suited for follow-up studies and treatment monitoring [64]. Visual evaluation is also sufficient to differentiate between different causes of pulmonary hypertension (PH) like PAH and chronic thromboembolic PH (CTEPH) [65]. Perfusion was diffusely reduced in PAH and focal defects occurred in CTEPH (Fig. 4, Fig. 5). In 45 patients with moderate to severe COPD, perfusion was matched to parenchymal alterations demonstrated by MDCT [66]. A high agreement on a lobar level was found between parenchymal destruction and reduction of perfusion in patients with severe emphysema (kappa of 0.8 ). In patients with cystic fibrosis the functional score, based on the visual evaluation of the perfusion defects, showed an acceptable intra- and interreader agreement (concordance correlation coefficient 0.89 and 0.8 , respectively) [67].

Semi-quantitative analysis of 3D perfusion data sets in eight healthy volunteers allowed for calculation of the leftto-right perfusion ratio (ratio 0.9 ), which was confirmed by phase-contrast flow measurements [68]. Furthermore, a shorter transit time and higher peak signal were found in the dorsal lung regions. Quantitative evaluation of perfusion was compared to perfusion scintigraphy for assessment of left-to-right perfusion ratios in 23 patients with various lung diseases [69]. The MR estimations of left-to-right perfusion ratios correlated significantly with those of perfusion scintigraphy scans $(P<0.01)$. The MR ratios computed from PBF showed the highest accuracy, followed by those from peak concentration and PBV.

One important clinical field for perfusion assessment is estimation of post-surgical lung function, for example, after lung cancer resection. So far, perfusion scintigraphy is routinely used for this purpose. In 60 patients with lung cancer, regional blood flow was assessed by scintigraphy and MR [34]. MR outperformed scintigraphy for assessment of postoperative FEV 1 ( $\mathrm{r}=0.93$ and $\mathrm{r}=0.89$, respectively). Therefore, it was concluded that perfusion MR is a feasible alternative to pulmonary perfusion scintigraphy for predicting postoperative lung function in patients with lung cancer.

Quantitative evaluation of different physiological effects was performed in nine healthy volunteers [70]. Examinations were performed in inspiratory and expiratory breathholds. A significantly higher perfusion was observed at expiratory breath-hold. Also a higher PBF was observed for the right lung. However, no ventro-dorsal perfusion gradient was observed, which was explained by the inclusion of central pulmonary vessels in the evaluation. After application of a vasodilatative agent (inhalation of $100 \%$ oxygen) a significant increase and redistribution of the pulmonary perfusion were found in ten healthy volunteers [71]. 
Fig. 4 Same patient as in Fig. 3. Absolute quantification of perfusion parameters

(a) pulmonary blood flow and (b) pulmonary blood volume. Especially the pulmonary blood flow nicely pronounces the area of the perfusion defect as well as the areas with reduced perfusion. Original perfusionweighted data set (presented as maximum intensity projection) See Fig. 3
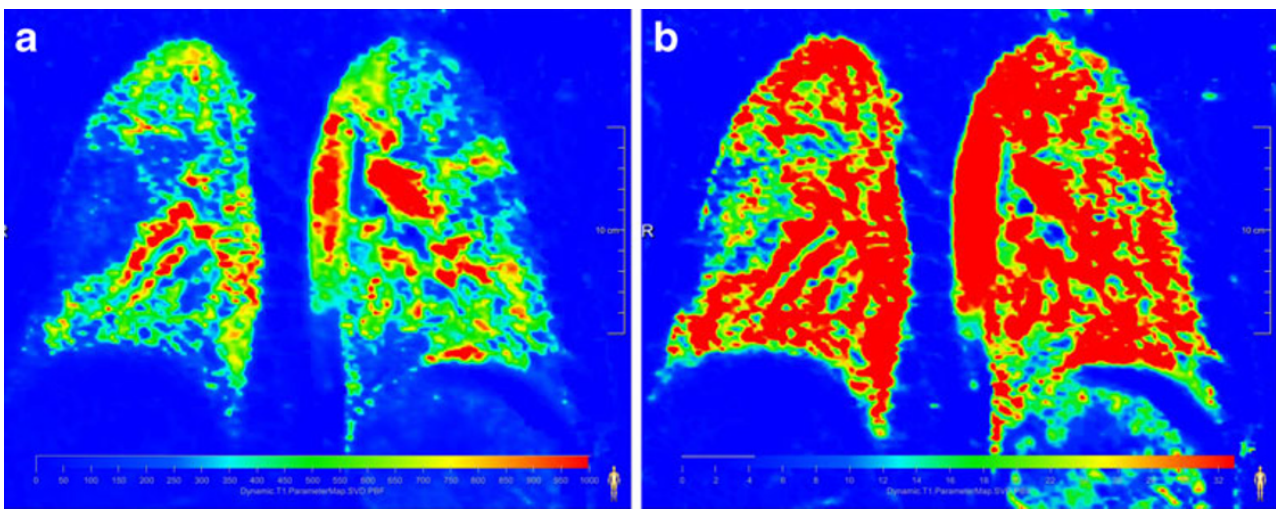

Therefore, MR perfusion seems to be suited for estimation of therapy response in patients with $\mathrm{PH}$ after administration of vasodilatative drugs.

In a group of 13 COPD patients, the pulmonary perfusion was found to be heterogeneously altered-PBF and PBV were significantly reduced and MTT prolonged [72].

Despite the pure quantification, another aspect of perfusion, although harder to establish, is the degree of homogeneity. In patients susceptible to high-altitude pulmonary oedema (HAPE-S) the perfusion inhomogeneity was compared between HAPE resistant volunteers and HAPE-S patients after $2 \mathrm{~h}$ of hypoxia (being equivalent to an altitude of $4,500 \mathrm{~m}$ ) [73]. Parameters indicating perfusion inhomogeneity increased during hypoxia in both populations, particularly in HAPE-S subjects where they increased significantly for almost all evaluations. These results were in accordance with those obtained by ASL technique [31]. Therefore, this kind of analysis, although not yet broadly used, may be important for future applications in diseases with peripheral vasoconstriction like pulmonary hypertension. So far, only conventional perfusion parameters have been assessed in patients with pulmonary hypertension. Compared to healthy, aged-matched volunteers, perfusion parameters are different in patients with pulmonary hypertension, with reduced PBF and PBV and increased MTT [74, 75]. Only the MTT showed a moderate linear relationship with MPAP $(r=0.54$
Fig. 5 Patient with pulmonary arterial hypertension

[(a) pulmonary blood flow and (b) pulmonary blood volume]. An overall reduced perfusion especially involving the lung periphery is noted. However, subpleural perfusion is still present and no wedge-shaped perfusion defects are seen (c) Original perfusion-weighted data set (presented as $10 \mathrm{~mm}$ maximum intensity projection)
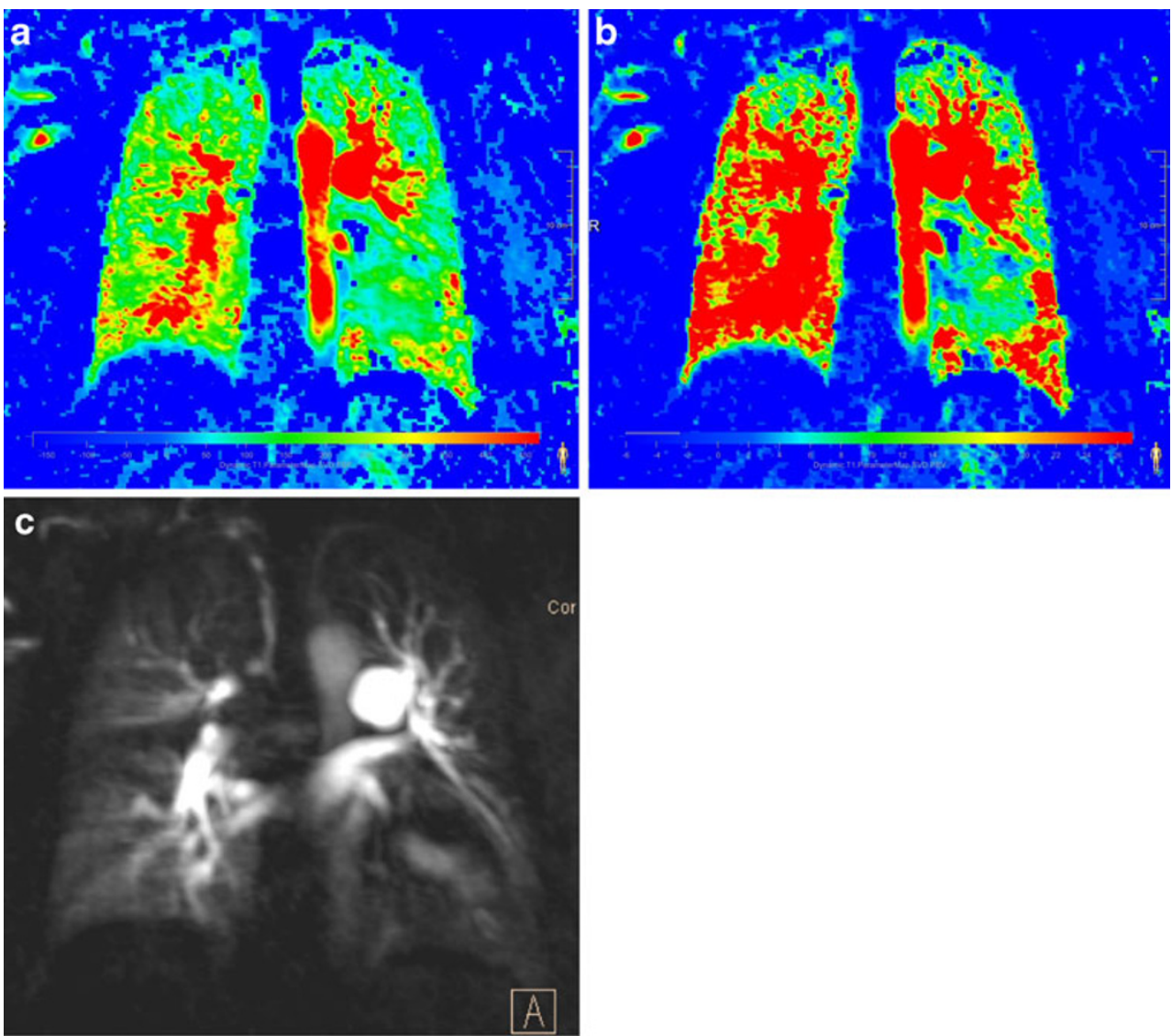
and $r=0.56$, respectively). Therefore, a severity assessment for pulmonary hypertension is currently not possible. It remains to be determined if perfusion MR might have a role for follow-up after treatment [58].

\section{Future developments}

Contrast-enhanced pulmonary perfusion can be routinely acquired on the present hardware. However the large data sets are difficult to handle and even visual evaluation might be challenging. Therefore, future developments needs to aim at providing easy-to-use post-processing utilities for visual and quantitative evaluation.

\section{Conclusion}

Non contrast-enhanced techniques for assessment of pulmonary perfusion are an important tool for understanding of lung physiology. Given the low spatial resolution and limited signal-to-noise ratio, a broad clinical application appears to be difficult. Contrast-enhanced perfusion techniques are broadly available and are easily and quickly performed, even in critical ill patients. Perfusion MR has shown good agreement with perfusion scintigraphy and SPECT. Therefore, this technique is ready to be used in a broader clinical area.

Acknowledgements The authors are grateful to Dr. Peter Kohlmann (Fraunhofer Mevis, Bremen, Germany) for providing the software used for generating the contrast-enhanced perfusion images. This review was partly supported by the Kompetenznetz Asthma/COPD (Competence Network Asthma/COPD) funded by the Federal Ministry of Education and Research (FKZ 01GI0881-0888).

\section{References}

1. Berthezène $Y$, Vexler V, Clément $O$, Mühler A, Moseley ME, Brasch RC (1992) Contrast-enhanced MR imaging of the lung: Assessment of ventilation and perfusion. Radiology 183:667-672

2. Attenberger UI, Ingrisch M, Busing K, Reiser M, Schoenberg SO, Fink C (2009) Magnetic resonance imaging of pulmonary perfusion. Technical requirements and diagnostic impact. Radiologe 49:739-747

3. Hopkins SR, Prisk GK (2010) Lung perfusion measured using magnetic resonance imaging: New tools for physiological insights into the pulmonary circulation. J Magn Reson Imaging 32:12871301

4. Martirosian P, Boss A, Schraml C, Schwenzer NF, Graf H, Claussen CD, Schick F (2010) Magnetic resonance perfusion imaging without contrast media. Eur J Nucl Med Mol Imaging online first:

5. Hatabu H, Tadamura E, Prasad PV, Chen Q, Buxton R, Edelman RR (2000) Noninvasive pulmonary perfusion imaging by STARHASTE sequence. Magn Reson Med 44:808-812

6. Roberts DA, Gefter WB, Hirsch JA, Rizi RR, Dougherty L, Lenkinski RE, Leigh JS Jr, Schnall MD (1999) Pulmonary perfusion: respiratory-triggered three-dimensional MR imaging with arterial spin tagging-preliminary results in healthy volunteers. Radiology 212:890-895

7. Lipson DA, Roberts DA, Hansen-Flaschen J, Gentile TR, Jones G, Thompson A, Dimitrov IE, Palevsky HI, Leigh JS, Schnall M, Rizi RR (2002) Pulmonary ventilation and perfusion scanning using hyperpolarized helium-3 MRI and arterial spin tagging in healthy normal subjects and in pulmonary embolism and orthotopic lung transplant patients. Magn Reson Med 47:1073-1076

8. Rizi RR, Lipson DA, Dimitrov IE, Ishii M, Roberts DA (2003) Operating characteristics of hyperpolarized $3 \mathrm{He}$ and arterial spin tagging in MR imaging of ventilation and perfusion in healthy subjects. Acad Radiol 10:502-508

9. Suga K, Ogasawara N, Okada M, Matsunaga N, Arai M (2002) Regional lung functional impairment in acute airway obstruction and pulmonary embolic dog models assessed with gadoliniumbased aerosol ventilation and perfusion magnetic resonance imaging. Invest Radiol 37:281-291

10. Ogasawara N, Suga K, Kawakami Y, Yamashita T, Zaki M, Matsunaga N (2004) Assessment of regional lung function impairment in airway obstruction and pulmonary embolic dogs with combined noncontrast electrocardiogram-gated perfusion and gadolinium diethylenetriaminepentaacetic acid aerosol magnetic resonance images. J Magn Reson Imaging 20:46-55

11. Martirosian P, Boss A, Fenchel M, Deimling M, Schafer J, Claussen CD, Schick F (2006) Quantitative lung perfusion mapping at $0.2 \mathrm{~T}$ using FAIR True-FISP MRI. Magn Reson Med 55:1065-1074

12. Bolar DS, Levin DL, Hopkins SR, Frank LF, Liu TT, Wong EC, Buxton RB (2006) Quantification of regional pulmonary blood flow using ASL-FAIRER. Magn Reson Med 55:1308-1317

13. Mai VM, Berr SS (1999) MR perfusion imaging of pulmonary parenchyma using pulsed arterial spin labeling techniques: FAIRER and FAIR. J Magn Reson Imaging 9:483-487

14. Pracht ED, Fischer A, Arnold JF, Kotas M, Flentje M, Jakob PM (2006) Single-shot quantitative perfusion imaging of the human lung. Magn Reson Med 56:1347-1351

15. Fischer A, Pracht ED, Arnold JF, Kotas M, Flentje M, Jakob PM (2008) Assessment of pulmonary perfusion in a single shot using SEEPAGE. J Magn Reson Imaging 27:63-70

16. Buxton RB, Frank LR, Wong EC, Siewert B, Warach S, Edelman RR (1998) A general kinetic model for quantitative perfusion imaging with arterial spin labeling. Magn Reson Med 40:383-396

17. Mai VM, Chen Q, Edelman RR (2000) Absolute quantification of pulmonary perfusion rates using flow-sensitive alternating inversion recovery with an extra radio frequency pulse (FAIRER)Proceedings of the 8th Annual Meeting of ISMRM,171

18. Brudin LH, Rhodes CG, Valind SO, Wollmer P, Hughes JM (1987) Regional lung density and blood volume in nonsmoking and smoking subjects measured by PET. J Appl Physiol 63:1324-1334

19. Wang T, Schultz G, Hebestreit H, Hebestreit A, Hahn D, Jakob PM (2003) Quantitative perfusion mapping of the human lung using 1H spin labeling. J Magn Reson Imaging 18:260-265

20. Lin YR, Wu MT, Huang TY, Tsai SY, Chung HW, Mai VM, Chen CY, Pan HB (2004) Comparison of arterial spin labeling and firstpass dynamic contrast-enhanced MR imaging in the assessment of pulmonary perfusion in humans: the inflow spin-tracer saturation effect. Magn Reson Med 52:1291-1301

21. Mai VM, Hagspiel KD, Christopher JM, Do HM, Altes T, KnightScott J, Stith AL, Maier T, Berr SS (1999) Perfusion imaging of the human lung using flow-sensitive alternating inversion recovery with an extra radiofrequency pulse (FAIRER). Magn Reson Imaging 17:355-361

22. Hopkins SR, Henderson AC, Levin DL, Yamada K, Arai T, Buxton RB, Prisk GK (2007) Vertical gradients in regional lung density and perfusion in the supine human lung: the Slinky effect. J Appl Physiol 103:240-248 
23. Henderson AC, Prisk GK, Levin DL, Hopkins SR, Buxton RB (2009) Characterizing pulmonary blood flow distribution measured using arterial spin labeling. NMR Biomed 22:1025-1035

24. Hopkins SR, Levin DL, Emami K, Kadlecek S, Yu J, Ishii M, Rizi RR (2007) Advances in magnetic resonance imaging of lung physiology. J Appl Physiol 102:1244-1254

25. Mai VM, Chen Q, Bankier AA, Blake M, Hagspiel KD, KnightScott J, Berr SS, Edelman RR (2001) Effect of lung inflation on arterial spin labeling signal in MR perfusion imaging of human lung. J Magn Reson Imaging 13:954-959

26. Fan L, Liu SY, Xiao XS, Sun F (2010) Demonstration of pulmonary perfusion heterogeneity induced by gravity and lung inflation using arterial spin labeling. Eur J Radiol 73:249-254

27. Levin DL, Buxton RB, Spiess JP, Arai T, Balouch J, Hopkins SR (2007) Effects of age on pulmonary perfusion heterogeneity measured by magnetic resonance imaging. J Appl Physiol 102:20642070

28. Keilholz SD, Knight-Scott J, Christopher JM, Mai VM, Berr SS (2001) Gravity-dependent perfusion of the lung demonstrated with the FAIRER arterial spin tagging method. Magn Reson Imaging 19:929-935

29. Keilholz SD, Mai VM, Berr SS, Fujiwara N, Hagspiel KD (2002) Comparison of first-pass Gd-DOTA and FAIRER MR perfusion imaging in a rabbit model of pulmonary embolism. J Magn Reson Imaging 16:168-171

30. Altes TA, Mai VM, Munger TM, Brookeman JR, Hagspiel KD (2005) Pulmonary embolism: comprehensive evaluation with MR ventilation and perfusion scanning with hyperpolarized helium-3, arterial spin tagging, and contrast-enhanced MRA. J Vasc Interv Radiol 16:999-1005

31. Hopkins SR, Garg J, Bolar DS, Balouch J, Levin DL (2005) Pulmonary blood flow heterogeneity during hypoxia and highaltitude pulmonary edema. Am J Respir Crit Care Med 171:83-87

32. Kluge A, Muller C, Hansel J, Gerriets T, Bachmann G (2004) Real-time MR with TrueFISP for the detection of acute pulmonary embolism: initial clinical experience. Eur Radiol 14:709-718

33. Fishman AP (1963) Hamilton WH, Dow P (eds) Dynamic of the Pulmonary Circulation. Handbook of Physiology, Sect. 2, Circulation, vol II., American Pysiological Society, Washington, DC, 1708

34. Ohno Y, Hatabu H, Higashino T, Takenaka D, Watanabe H, Nishimura Y, Yoshimura M, Sugimura K (2004) Dynamic perfusion MRI versus perfusion scintigraphy: prediction of postoperative lung function in patients with lung cancer. AJR Am J Roentgenol 182:7378

35. Fink C, Ley S, Kroeker R, Requardt M, Kauczor HU, Bock M (2005) Time-resolved contrast-enhanced three-dimensional magnetic resonance angiography of the chest: combination of parallel imaging with view sharing (TREAT). Invest Radiol 40:40-48

36. Korosec F, Frayne R, Grist T, Mistretta C (1996) Time-resolved contrast-enhanced 3D MR angiography. Magn Reson Med $36: 345-351$

37. Fink C, Puderbach M, Ley S, Zaporozhan J, Plathow C, Kauczor HU (2005) Time-resolved echo-shared parallel MRA of the lung: observer preference study of image quality in comparison with non-echo-shared sequences. Eur Radiol 15:2070-2074

38. Fink C, Bock M, Puderbach M, Schmähl A, Delorme S (2003) Partially parallel three-dimensional magnetic resonance imaging for the assessment of lung perfusion - initial results. Invest Radiol 38:482-488

39. Attenberger UI, Ingrisch M, Dietrich O, Herrmann K, Nikolaou K, Reiser MF, Schonberg SO, Fink C (2009) Time-resolved 3D pulmonary perfusion MRI: comparison of different k-space acquisition strategies at 1.5 and $3 \mathrm{~T}$. Invest Radiol 44:525-531
40. Fink C, Bock M, Kiessling F, Lichy MP, Krissak R, Zuna I, Schmahl A, Delorme S, Kauczor HU (2005) Time-resolved contrast-enhanced three-dimensional pulmonary MR-angiography: $1.0 \mathrm{M}$ gadobutrol vs. 0.5M gadopentetate dimeglumine. J Magn Reson Imaging 19:202208

41. Kreitner KF, Kunz RP, Weschler C, Ley S, Krummenauer F, Schreiber WG, Thelen M (2005) Analysis of first-pass bolus geometry in contrast-enhanced MRA of thoracic vessels. Fortschr Röntgenstr 177:646-654

42. Fink C, Risse F, Semmler W, Schoenberg SO, Kauczor HU, Reiser MF (2006) MRI of pulmonary perfusion. Radiologe 46:290-299

43. Risse F, Fink C, Ley S, Kauczor HU, Schad LR (2004) Perfusion quantification of the whole lung using singular value decomposition with optimized threshold. Proc Intl Soc Mag Med 11:2611

44. Meier P, Zierler KL (1954) On the theory of the indicator-dilution method for measurement of blood flow and volume. J Appl Physiol 6:731-744

45. Ostergaard L, Weisskoff RM, Chesler DA, Gyldensted C, Rosen BR (1996) High resolution measurement of cerebral blood flow using intravascular tracer bolus passages. Part I: Mathematical approach and statistical analysis. Magn Reson Med 36:715-725

46. Ostergaard L, Sorensen AG, Kwong KK, Weisskoff RM, Gyldensted C, Rosen BR (1996) High resolution measurement of cerebral blood flow using intravascular tracer bolus passages. Part II: Experimental comparison and preliminary results. Magn Reson Med 36:726-736

47. Risse F, Kuder TA, Kauczor HU, Semmler W, Fink C (2009) Suppression of pulmonary vasculature in lung perfusion MRI using correlation analysis. Eur Radiol 19:2569-2575

48. Bock M, Schoenberg SO, Floemer F, Schad LR (2000) Separation of arteries and veins in 3D MR angiography using correlation analysis. Magn Reson Med 43:481-487

49. Santini F, Patil S, Meckel S, Scheffler K, Wetzel SG (2008) Double-reference cross-correlation algorithm for separation of the arteries and veins from 3D MRA time series. J Magn Reson Imaging 28:646-654

50. Kostler H, Ritter C, Lipp M, Beer M, Hahn D, Sandstede J (2004) Prebolus quantitative MR heart perfusion imaging. Magn Reson Med 52:296-299

51. Risse F, Semmler W, Kauczor HU, Fink C (2006) Dual-bolus approach to quantitative measurement of pulmonary perfusion by contrast-enhanced MRI. J Magn Reson Imaging 24:1284-1290

52. Puderbach M, Risse F, Biederer J, Ley-Zaporozhan J, Ley S, Szabo G, Semmler W, Kauczor HU (2008) In vivo Gd-DTPA concentration for MR lung perfusion measurements: Assessment with computed tomography in a porcine model. Eur Radiol 18:2102-2107

53. Nikolaou K, Schoenberg SO, Brix G, Goldman JP, Attenberger U, Kuehn B, Dietrich O, Reiser MF (2004) Quantification of pulmonary blood flow and volume in healthy volunteers by dynamic contrast-enhanced magnetic resonance imaging using a parallel imaging technique. Invest Radiol 39:537-545

54. Schuster DP, Kaplan JD, Gauvain K, Welch MJ, Markham J (1995) Measurement of regional pulmonary blood flow with PET. J Nucl Med 36:371-377

55. Brudin LH, Valind SO, Rhodes CG, Turton DR, Hughes JM (1986) Regional lung hematocrit in humans using positron emission tomography. J Appl Physiol 60:1155-1163

56. Ohno $Y$, Murase K, Higashino T, Nogami M, Koyama H, Takenaka D, Kawamitu H, Matsumoto S, Hatabu H, Sugimura K (2007) Assessment of bolus injection protocol with appropriate concentration for quantitative assessment of pulmonary perfusion by dynamic contrast-enhanced MR imaging. J Magn Reson Imaging 25:55-65

57. Ingrisch M, Dietrich O, Attenberger UI, Nikolaou K, Sourbron S, Reiser MF, Fink C (2010) Quantitative pulmonary perfusion 
magnetic resonance imaging: influence of temporal resolution and signal-to-noise ratio. Invest Radiol 45:7-14

58. Ley S, Gruenig E, Risse F, Ehlken N, Mereles D, Kauczor H-U (2008) Treatment monitoring in patients with pulmonary arterial hypertension (PAH) using quantitative 3D MR pulmonary perfusionECRB-818

59. Ley-Zaporozhan J, Molinari F, Risse F, Puderbach M, Schenk J-P, Kopp-Schneider A, Kauczor H-U, Ley S (2010) Repeatability and reproducibility of quantitative whole-lung perfusion MRI. J Thorac Imaging in press

60. Bottger T, Grunewald K, Schobinger M, Fink C, Risse F, Kauczor HU, Meinzer HP, Wolf I (2007) Implementation and evaluation of a new workflow for registration and segmentation of pulmonary MRI data for regional lung perfusion assessment. Phys Med Biol 52:1261-1275

61. Fink C, Ley S, Puderbach M, Plathow C, Bock M, Kauczor HU (2004) 3D pulmonary perfusion MRI and MR angiography of pulmonary embolism in pigs after a single injection of a blood pool MR contrast agent. Eur Radiol 14:1291-1296

62. Kluge A, Gerriets T, Stolz E, Dill T, Mueller KD, Mueller C, Bachmann G (2006) Pulmonary perfusion in acute pulmonary embolism: agreement of MRI and SPECT for lobar, segmental and subsegmental perfusion defects. Acta Radiol 47:933-940

63. Ohno Y, Koyama H, Matsumoto K, Onishi Y, Nogami M, Takenaka D, Yoshikawa T, Matsumoto S, Sugimura K (2010) Dynamic MR perfusion imaging: capability for quantitative assessment of disease extent and prediction of outcome for patients with acute pulmonary thromboembolism. J Magn Reson Imaging 31:1081-1090

64. Fink C, Ley S, Schoenberg SO, Reiser MF, Kauczor HU (2007) Magnetic resonance imaging of acute pulmonary embolism. Eur Radiol 17:2546-2553

65. Ley S, Fink C, Zaporozhan J, Borst MM, Meyer FJ, Puderbach M, Eichinger M, Plathow C, Grunig E, Kreitner KF, Kauczor HU (2005) Value of high spatial and high temporal resolution magnetic resonance angiography for differentiation between idiopathic and thromboembolic pulmonary hypertension: initial results. Eur Radiol 15:2256-2263

66. Ley-Zaporozhan J, Ley S, Eberhardt R, Weinheimer O, Fink C, Puderbach M, Eichinger M, Herth F, Kauczor HU (2007) Assessment of the relationship between lung parenchymal destruction and impaired pulmonary perfusion on a lobar level in patients with emphysema. Eur J Radiol 63:76-83

67. Eichinger M, Optazaite DE, Kopp-Schneider A, Hintze C, Biederer J, Niemann A, Mall MA, Wielputz MO, Kauczor HU, Puderbach M
(2011) Morphologic and functional scoring of cystic fibrosis lung disease using MRI. Eur J Radiol in press:

68. Ley S, Fink C, Puderbach M, Plathow C, Risse F, Kreitner KF, Kauczor HU (2004) Contrast-enhanced 3D MR perfusion of the lung: application of parallel imaging technique in healthy subjects. Fortschr Röntgenstr 176:330-334

69. Molinari F, Fink C, Risse F, Tuengerthal S, Bonomo L, Kauczor HU (2006) Assessment of differential pulmonary blood flow using perfusion magnetic resonance imaging: comparison with radionuclide perfusion scintigraphy. Invest Radiol 41:624-630

70. Fink C, Ley S, Risse F, Eichinger M, Zaporozhan J, Buhmann R, Puderbach M, Plathow C, Kauczor HU (2005) Effect of inspiratory and expiratory breathhold on pulmonary perfusion: assessment by pulmonary perfusion magnetic resonance imaging. Invest Radiol 40:72-79

71. Ley S, Puderbach M, Risse F, Ley-Zaporozhan J, Eichinger M, Takenaka D, Kauczor HU, Bock M (2007) Impact of oxygen inhalation on the pulmonary circulation: assessment by magnetic resonance (MR)-perfusion and MR-flow measurements. Invest Radiol 42:283-290

72. Ohno Y, Hatabu H, Murase K, Higashino T, Kawamitsu H, Watanabe H, Takenaka D, Fujii M, Sugimura K (2004) Quantitative assessment of regional pulmonary perfusion in the entire lung using threedimensional ultrafast dynamic contrast-enhanced magnetic resonance imaging: Preliminary experience in 40 subjects. J Magn Reson Imaging 20:353-365

73. Dehnert C, Risse F, Ley S, Kuder TA, Buhmann R, Puderbach M, Menold E, Mereles D, Kauczor HU, Bartsch P, Fink C (2006) Magnetic resonance imaging of uneven pulmonary perfusion in hypoxia in humans. Am J Respir Crit Care Med 174:11321138

74. Ohno Y, Hatabu H, Murase K, Higashino T, Nogami M, Yoshikawa T, Sugimura K (2007) Primary pulmonary hypertension: 3D dynamic perfusion MRI for quantitative analysis of regional pulmonary perfusion. AJR Am J Roentgenol 188:48-56

75. Ley S, Mereles D, Risse F, Grunig E, Ley-Zaporozhan J, Tecer Z, Puderbach M, Fink C, Kauczor HU (2007) Quantitative 3D pulmonary MR-perfusion in patients with pulmonary arterial hypertension: Correlation with invasive pressure measurements. Eur J Radiol 61:251-255

76. Kluge A, Luboldt W, Bachmann G (2006) Acute pulmonary embolism to the subsegmental level: diagnostic accuracy of three MRI techniques compared with 16-MDCT. AJR Am J Roentgenol 187:W7-W14 